\title{
Di-Higgs resonance searches in weak boson fusion
}

\author{
Rahool Kumar Barman, ${ }^{1, *}$ Christoph Englert ${ }^{2, \dagger}$ Dorival Gonçalves $\odot,{ }^{3, *}$ and Michael Spannowsky ${ }^{4, \S}$ \\ ${ }^{1}$ School of Physical Sciences, Indian Association for the Cultivation of Science, Kolkata 700032, India \\ ${ }^{2}$ School of Physics and Astronomy, University of Glasgow, Glasgow G12 8QQ, United Kingdom \\ ${ }^{3}$ Department of Physics, Oklahoma State University, Stillwater, Oklahoma 74078, USA \\ ${ }^{4}$ Institute for Particle Physics Phenomenology, Department of Physics, Durham University, \\ Durham DH1 3LE, United Kingdom
}

(Received 28 July 2020; accepted 24 August 2020; published 17 September 2020)

\begin{abstract}
The search for di-Higgs final states is typically limited at the LHC to the dominant gluon fusion channels, with weak boson fusion only assuming a spectator role. In this work, we demonstrate that when it comes to searches for resonant structures that arise from isosinglet mixing in the Higgs sector, the weak boson fusion sideline can indeed contribute to winning the discovery game. Extending existing experimental resonance searches by including both contributions is therefore crucial.
\end{abstract}

DOI: 10.1103/PhysRevD.102.055014

\section{INTRODUCTION}

The search for new physics beyond the Standard Model (SM) is a key pillar of the Large Hadron Collider (LHC) physics program. As significant deviations from the SM expectation have remained elusive after the Higgs boson's discovery so far, the nature of the electroweak scale is still fundamentally unknown. A particularly relevant process in this context is the production of multiple Higgs bosons. First, multi-Higgs production directly probes aspects of spontaneous symmetry breaking that cannot be accessed with weak boson or heavy quark physics. Second, the inclusive production cross section of Higgs pairs of around $30 \mathrm{fb}$ [1-7] is about 3 orders of magnitude smaller than single Higgs production, thus highlighting the statistical difficulty that experimental investigations face in this area.

Multi-Higgs production is phenomenologically limited to Higgs pairs [8], at least in the near future [9,10], and as with single Higgs production, gluon fusion (GF) contributes to the bulk of the production cross section. While Higgs production via weak boson fusion (WBF) with its distinct phenomenological properties [11-14] and large cross section plays an important role in the investigation of the Higgs boson's properties, di-Higgs production from weak boson fusion will be statistically limited at the LHC

\footnotetext{
*psrkb2284@iacs.res.in

christoph.englert@glasgow.ac.uk

*dorival@okstate.edu

${ }^{\S}$ michael.spannowsky@durham.ac.uk
}

Published by the American Physical Society under the terms of the Creative Commons Attribution 4.0 International license. Further distribution of this work must maintain attribution to the author(s) and the published article's title, journal citation, and DOI. Funded by SCOAP ${ }^{3}$.
[15-17]. WBF-type analyses are further hampered by the importance of the top threshold for gluon fusion production [18] and the necessity to relax central jet vetos to retain a reasonable WBF signal count through central $h \rightarrow b \bar{b}$ decays. Experimental analyses typically mitigate the nonapplicability of central jet vetos in the WBF selection by considering stringent invariant jet pair masses; see, e.g., Ref. [19]. While such a selection serves to purify signal samples toward the WBF component, forward jets will also arise from gluon fusion samples [20-23] when biased toward valence quark-flavored initial state processes and the question of the size of the potential, model-dependent GF component remains.

Resonant phenomena in weak boson fusion are less studied from a phenomenological perspective than their GF counterparts. A bias toward GF-like production is understandable as two-Higgs-doublet extensions of the SM in particular as prototypes of supersymmetric theories lead to gauge-phobic scalars, and WBF production of exotic states, e.g., the additional $C P$-odd scalar proceeds dominantly through GF. However, the observation of resonances in WBF would have exciting theoretical implications. Introducing a new resonant beyond the Standard Model scalar in the WBF modes rests on nonalignment [24], $C P$ violation [25], a significant nondoublet component of the electroweak vacuum (e.g., [26-28]), or combinations of these.

Electroweak symmetry breaking from triplets faces a theoretical reservation related to the fine-tuning of the rho parameter [29]. ${ }^{1}$ Phenomenologically, (tree-level) custodial triplet extensions lead to a range of additional exotic final

\footnotetext{
${ }^{1}$ It remains as a possibility of strong electroweak symmetry breaking in realistic UV constructions [30,31].
} 
states, most notably a doubly charged Higgs that is predominantly produced through weak boson fusion as part of a fermiophobic custodial quintet [32-36]. Electrically uncharged components of the custodial triplet will not decay promptly to the $125 \mathrm{GeV}$ state if the latter is identified as a doubletlike state, again due to custodial isospin. $C P$ violation is typically a small effect in actual scans [25] such that a competitive production through WBF is typically suppressed.

The possibility of nonalignment (i.e., the physical $125 \mathrm{GeV}$ Higgs boson not being fully aligned with fluctuations around the electroweak vacuum) remains as an a priori relevant parameter space for WBF to be relevant. The mixing of isospin singlet states is present in any Higgs sector extension but most transparently analyzed in the so-called Higgs portal scenario [37]. This model also fully correlates the exotic Higgs production with observed $m_{h} \simeq 125 \mathrm{GeV}$ Higgs boson phenomenology, which turns any sensitivity projection for heavy Higgs states into a conservative estimate as new, nonsinglet fields will loosen the tight correlations of the singlet extensions.

The relevance of WBF production is further highlighted in singlet scenarios by the fact that for SM Higgs-like states with masses $\mathcal{O}(\mathrm{TeV}), \mathrm{GF}$ and WBF productions become comparable $[38,39]$. This strongly indicates that if such a state is realized in nature, both GF and WBF play a priori an equally important role in the discovery of new physics. As there is accidental destructive interference of $p p \rightarrow H \rightarrow t \bar{t}$ with QCD continuum top pair production [40-46] which particularly affects the sensitivity in the singlet-extension scenario [47], gaining sensitivity in the $H \rightarrow h h$ decays is not only necessary but also possibly the only phenomenological robust avenue to successfully detect such scenarios. Depending on the Higgs potential, these channels might be favored over the decays into massive electroweak gauge bosons, which are additional relevant channels.

In this work, we perform a detailed investigation of WBF production of exotic Higgs bosons $p p \rightarrow H j j$ arising from isosinglet mixing, in particular in their decay $H \rightarrow h h$. We include the gluon fusion component keeping the full $m_{t}$ dependence and highlight the interplay of both production modes and their relevance to hone the discovery potential at the LHC. In particular, we show that gluon fusion remains phenomenologically relevant and should therefore be reflected as an appropriate signal contribution in any analysis that seeks to inform further theoretical investigations.

We organize this paper as follows: In Sec. II, we provide a short summary of the key phenomenological aspects of the singlet-extension scenario, which acts as the vehicle of this work. We stress that our findings readily generalize to more complex scenarios. Section II is devoted to the WBF di-Higgs resonance analysis. We conclude in Sec. IV.

\section{THE MODEL}

We consider the extension of the SM with Higgs doublet $\Phi_{s}$ by an additional singlet $\Phi_{h}$ under the SM gauge group
$V=\mu_{s}^{2}\left|\Phi_{s}\right|^{2}+\lambda_{s}\left|\Phi_{s}\right|^{4}+\mu_{h}^{2}\left|\Phi_{h}\right|^{2}+\lambda_{h}\left|\Phi_{h}\right|^{4}+\eta\left|\Phi_{s}\right|^{2}\left|\Phi_{h}\right|^{2}$.

Expanding around the vacuum expectation values of the respective fields

$$
v_{i}^{2}=\frac{1}{\lambda_{i}}\left(-\mu_{i}^{2}-\frac{\eta}{2} v_{j \neq i}^{2}\right), \quad i, j=s, h
$$

via $\Phi_{i}=\left(v_{i}+H_{i}\right) / \sqrt{2}$ leads to a mixing of Lagrangian eigenstates in the mass basis

$$
\begin{aligned}
h & =\cos \theta H_{s}+\sin \theta H_{h}, \\
H & =-\sin \theta H_{s}+\cos \theta H_{h} .
\end{aligned}
$$

We will implicitly identify $h$ with the observed lighter $m_{h} \simeq$ $125 \mathrm{GeV}$ boson aligned with the SM expectation; i.e., we will be particularly interested in the region $\cos \theta \lesssim 1$. The masses are given by

$m_{h, H}^{2}=\left(\lambda_{s} v_{s}^{2}+\lambda_{h} v_{h}^{2}\right) \mp \sqrt{\left(\lambda_{s} v_{s}^{2}-\lambda_{h} v_{h}^{2}\right)^{2}+\eta^{2} v_{s}^{2} v_{h}^{2}}$

and

$$
\tan 2 \theta=\frac{\eta v_{s} v_{h}}{\lambda_{s} v_{s}^{2}-\lambda_{h} v_{h}^{2}},
$$

while $v_{s} \simeq 246 \mathrm{GeV}$ from electroweak symmetry breaking in the SM.

We assume no additional decay channels, which means that signal strengths of the SM-like Higgs are modified $\mu=\cos ^{2} \theta . H$ boson production cross sections as a function of $m_{H}$ can be obtained from the SM ones [38] by rescaling with $\sin ^{2} \theta$; branching ratios are unmodified for $m_{H}<2 m_{h}$. We are particularly interested in the region $m_{H} \geq 2 m_{h}$ where cascade decays $H \rightarrow h h$ are open. In this case, the heavy Higgs partner receives a leading order additional contribution to its decay width

$$
\Gamma(H \rightarrow h h)=\frac{c_{H h h}^{2}}{32 m_{H} \pi} \sqrt{1-\frac{4 m_{h}^{2}}{m_{H}^{2}}}
$$

with

$$
\begin{aligned}
c_{H h h}= & 3 \sin 2 \theta\left(\lambda_{s} v_{s} \cos \theta-\lambda_{h} v_{h} \sin \theta\right)-\tan 2 \theta\left(\lambda_{s} v_{s}^{2}-\lambda_{h}^{2} v_{h}^{2}\right) \\
& \times\left[\left(1-3 \cos ^{2} \theta\right) \frac{\sin \theta}{v_{h}}-\left(1-3 \sin ^{2} \theta\right) \frac{\cos \theta}{v_{s}}\right] .
\end{aligned}
$$

The potential measurement of $\Gamma(H \rightarrow h h)$ together with the masses $m_{h, H}$ and SM signal strength and weak boson masses allows us to fully reconstruct the singlet-extended Higgs potential. A range of precision computations from a QCD and electroweak point of view have become available 
recently [48-52] with strongest constraints typically arising from the $W$ mass measurement $[51,53]$.

\section{ANALYSIS}

We derive the LHC sensitivity to di-Higgs resonances in the vector boson fusion (VBF) channel $p p \rightarrow H j j$, with $H \rightarrow h h \rightarrow 4 b$. The signal is characterized by four bottom tagged jets in association with two light-flavor jets. The leading backgrounds for this process are $p p \rightarrow 4 b+2 j$, $2 b+4 j$, and $t \bar{t} b \bar{b}$.

We generate the WBF and QCD $p p \rightarrow(H \rightarrow h h) j j$ signal samples with VBFNLO [54], which we have modified to include the $H \rightarrow h h$ decay. The backgrounds are generated with MADGRAPH5AMC@NLO [55]. All samples are generated at leading order with center-of-mass energy of $\sqrt{s}=13 \mathrm{TeV}$. Parton shower, hadronization, and underlying event effects are accounted for with PYTHIA8 [56]. Jets are defined through the anti- $k_{T}$ algorithm with $R=0.4$, $p_{T j}>30 \mathrm{GeV}$, and $\left|\eta_{j}\right|<4.5$ via FastJet [57]. We assume $70 \% b$-tagging efficiency and a $1 \%$ mistag rate.

We start our analysis demanding at least six jets in the final state, where four of those are $b$ tagged. We impose a minimum threshold for the invariant mass for the four $b$ jets of $m_{4 b}>350 \mathrm{GeV}$ and veto leptons with $p_{T \ell}>12 \mathrm{GeV}$ and $\left|\eta_{\ell}\right|<2.5$. The two light-flavor jets with highest rapidity $j_{1,2}$ satisfy the VBF topology falling in different hemispheres of the detector $\eta_{j 1} \times \eta_{j 2}<0$, with large rapidity separation $\left|\eta_{j 1}-\eta_{j 2}\right|>4.2$, and sizable invariant mass $m_{j j}>1 \mathrm{TeV}$.

While the WBF signal displays suppressed extra jet emissions in the central region of the detector, the bulk of the QCD background radiation is centered around this regime [58-61]. In Fig. 1, we illustrate this property displaying two mass scenarios for the WBF signal samples, $m_{H}=0.5$ and $1 \mathrm{TeV}$. The more massive the signal resonance is, the further forward the tagging jets hit the

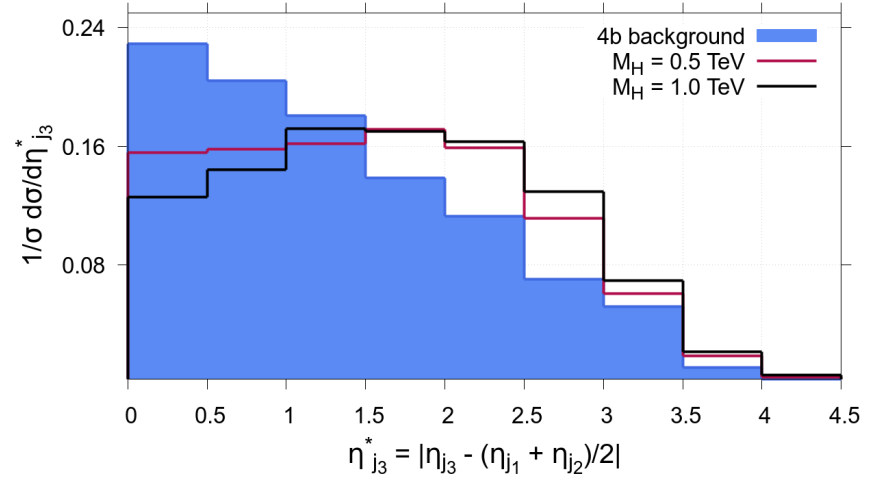

FIG. 1. Normalized distribution of $\eta_{j_{3}}^{\star}=\left|\eta_{j 3}-\left(\eta_{j 1}+\eta_{j 2}\right) / 2\right|$ for the dominant $4 b$ background (blue) and the WBF signal events $M_{H}=0.5 \mathrm{TeV}$ (red) and $1 \mathrm{TeV}$ (black) after imposing the basic selection cuts and the VBF selections: $\eta_{j 1} \times \eta_{j 2}<0$, $\left|\eta_{j 1}-\eta_{j 2}\right|>4.2$, and $m_{j j}>1 \mathrm{TeV}$. detector. This phenomenological pattern is related to gauge boson scattering $V V \rightarrow h h$ around the heavy Higgs pole, where the longitudinal and transverse scattering amplitudes scale as $\mathcal{A}_{L L} / \mathcal{A}_{T T} \sim m_{H}^{2} / m_{V}^{2}$ for $m_{H} \gg m_{V}$ [62-64]. We explore this feature to further suppress the backgrounds imposing that the rapidity for the third jet $\eta_{j 3}$ satisfies the relation

$$
\left|\eta_{j 3}-\frac{\eta_{j 1}+\eta_{j 2}}{2}\right|>2.5
$$

After establishing the VBF topology, the next step of the analysis focuses on the Higgs boson's reconstruction. This is performed by identifying among the four $b$ jets the pair whose invariant mass $m_{h 1}$ is closest to the Higgs mass, $m_{h}=125 \mathrm{GeV}$. The remaining $b$-jet pair defines the second Higgs boson candidate $h_{2}$. In the two-dimensional space defined by the masses of the Higgs boson candidates $\left(m_{h 1}, m_{h 2}\right)$, the signal region is defined to be within the circular region

$$
\sqrt{\left(\frac{m_{h 1}-125 \mathrm{GeV}}{20 \mathrm{GeV}}\right)^{2}+\left(\frac{m_{h 2}-125 \mathrm{GeV}}{20 \mathrm{GeV}}\right)^{2}}<1 .
$$

To further improve the $m_{4 b}$ mass resolution, each Higgs boson candidate's four-momentum is scaled by the correction factor $m_{h} / m_{h 1(2)}$. This improves the signal $m_{4 b}$ resolution from $20 \%$ to $40 \%$, depending on the heavy Higgs mass hypothesis, and presents subleading effects to the background $m_{4 b}$ distribution [65].

Since very few multijet background events pass the cutflow analysis with large $m_{4 b}$, we follow a similar statistical procedure performed by the ATLAS Collaboration in their $p p \rightarrow H \rightarrow h h \rightarrow 4 b$ study [66]. Namely, the statistical precision for the $m_{4 b}$ distribution at high energies is improved by fitting the background distribution at low invariant masses $m_{4 b}<1 \mathrm{TeV}$ with the functional form

$$
F\left(m_{4 b}\right)=a \frac{s}{m_{4 b}^{2}}\left(1-\frac{m_{4 b}}{\sqrt{s}}\right)^{b-c \log \frac{m_{4 b}}{\sqrt{s}}}
$$

where $a, b$, and $c$ are real free parameters and $\sqrt{s}$ the LHC center-of-mass energy. This also emulates a data-driven approach that is typically the method of choice when backgrounds are only poorly understood from a systematic and theoretical perspective; see, e.g., [67,68]. As we are looking for a resonance on top of a steeply falling background, such a method provides a particularly motivated approach to reduce uncertainties.

In Fig. 2, we illustrate the invariant mass distribution $m_{4 b}$ for the signal and background components after the full cutflow analysis shown in Table I. While the WBF signal component displays dominant contributions to the event rate, the VBF GF signal can result in non-negligible 


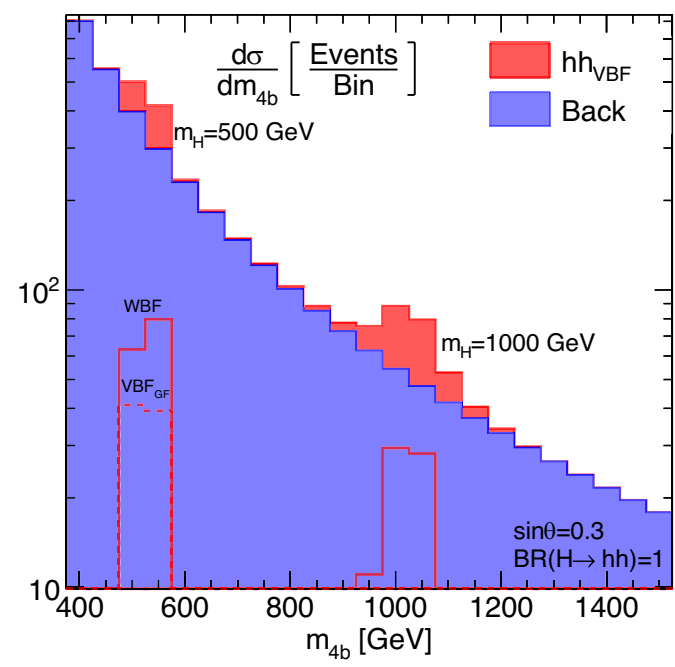

FIG. 2. Stacked $m_{4 b}$ distribution for the signal and background events after the complete cut-flow analysis shown in Table I. The VBF signal hypotheses are also shown in the nonstacked format with the WBF (solid line) and GF (dashed line) components independently displayed. We assume $\operatorname{BR}(H \rightarrow h h)=1$ and $\sin \theta=0.3$ with the LHC running at $\sqrt{s}=13 \mathrm{TeV}$ and integrated luminosity $\mathcal{L}=3 \mathrm{ab}^{-1}$.

additions to the event count. It should be noted that the larger the signal mass $m_{H}$ is, the larger the relative WBF component becomes.

To estimate the High Luminosity (HL)-LHC sensitivity to the resonant VBF $h h$ signal, we calculate a binned loglikelihood analysis based on the $m_{4 b}$ distribution using the $\mathrm{CL}_{s}$ method [73]. We assume the integrated luminosity $\mathcal{L}=3 \mathrm{ab}^{-1}$. In Fig. 3, we present the 95\% C.L. sensitivity to the heavy Higgs-singlet mixing $\sin \theta$ as a function of the heavy Higgs boson mass $m_{H}$. Motivated by the Goldstone boson equivalence theorem for $m_{H} \gg m_{W}$, we assume the heavy Higgs branching ratio to di-Higgs $\operatorname{BR}(H \rightarrow h h)=1 / 4$. To illustrate the importance of the VBF GF signal component, we separately show the signal sensitivity accounting for the full VBF sample and only for

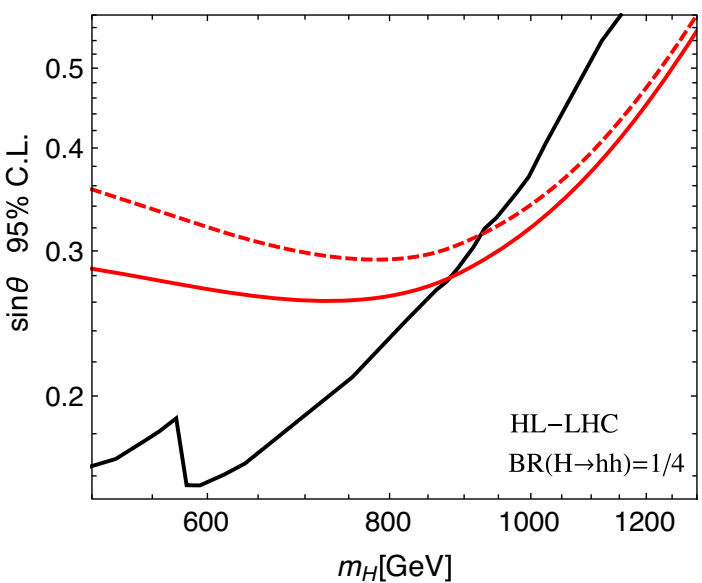

FIG. 3. $95 \%$ C.L. limit on the Higgs-singlet mixing as a function of the heavy Higgs boson mass $m_{H}$. We show both the VBF $p p \rightarrow H j j \rightarrow 4 b j j$ (red solid) and GF $p p \rightarrow H \rightarrow 4 b$ (black) limits. To estimate the importance of the VBF GF signal component to the $\mathrm{VBF}$ analysis, we also show the bound considering only the WBF signal component (red dashed). We assume the heavy Higgs boson branching ratio to di-Higgs $\operatorname{BR}(H \rightarrow h h)=1 / 4$ and the LHC at $13 \mathrm{TeV}$ with integrated luminosity $\mathcal{L}=3 \mathrm{ab}^{-1}$.

its WBF component. We observe that the VBF GF results in non-negligible contributions for the low mass regime $500 \mathrm{GeV}<m_{H}<900 \mathrm{GeV}$.

To compare our new VBF di-Higgs resonance search with the existing limits, we use the CMS $p p \rightarrow H \rightarrow h h \rightarrow$ $4 b$ study [65]. CMS derives the 95\% C.L. limit on the heavy Higgs cross section $\sigma(p p \rightarrow H \rightarrow h h \rightarrow 4 b)$ as a function of its mass $m_{H}$. We translate this bound in terms of the mixing $\sin \theta$ in Fig. 3, using the heavy Higgs production cross section at the next-to-next-to-leading order (NNLO) + next-to-next-to-leading logarithmic (NNLL) QCD, including top and bottom quark mass effects up to NLO $[4,38,74]$. The CMS limit on the heavy Higgs cross section was scaled to the HL-LHC integrated luminosity, $\mathcal{L}=3 \mathrm{ab}^{-1}$. The discontinuity on the CMS limit

TABLE I. Cut-flow table showing the cross section (in fb) for the VBF signal and backgrounds. The VBF signal is decomposed between the WBF and GF components. The background rates are normalized by the next-to-leadingorder (NLO) K factors: $1.7(4 b)$ [55], $1.3(2 b 2 j)$ [55], and $1.8(t \bar{t} b \bar{b})$ [69]. The signal rate is given with $\mathrm{BR}(H \rightarrow$ $h h)=1$ and $\sin \theta=0.3$. The GF signal rates are also normalized by the NLO K factor: 1.65. QCD corrections for the WBF process are included through an appropriate scale choice [70] and through MCFM for the gluon fusion contribution employing the heavy top limit $[71,72]$.

\begin{tabular}{lccc}
\hline \hline Process & Basic selections & VBF topology & Double Higgs reconstruction \\
\hline $4 b$ & 250 & 47 & 1.2 \\
$2 b 2 j$ & $4.9 \times 10^{-1}$ & $1.0 \times 10^{-1}$ & $\cdots$ \\
$t \bar{t} b \bar{b}$ & 90 & 3.7 & $3.0 \times 10^{-3}$ \\
WBF $m_{H}=500 \mathrm{GeV}$ & $2.6 \times 10^{-1}$ & $1.3 \times 10^{-1}$ & $5.0 \times 10^{-2}$ \\
$\mathrm{GF} m_{H}=500 \mathrm{GeV}$ & $2.2 \times 10^{-1}$ & $7.1 \times 10^{-2}$ & $2.8 \times 10^{-2}$ \\
$\mathrm{WBF} m_{H}=1 \mathrm{TeV}$ & $9.4 \times 10^{-2}$ & $5.4 \times 10^{-2}$ & $3.2 \times 10^{-2}$ \\
$\mathrm{GF} m_{H}=1 \mathrm{TeV}$ & $2.2 \times 10^{-2}$ & $8.3 \times 10^{-3}$ & $4.7 \times 10^{-3}$ \\
\hline \hline
\end{tabular}




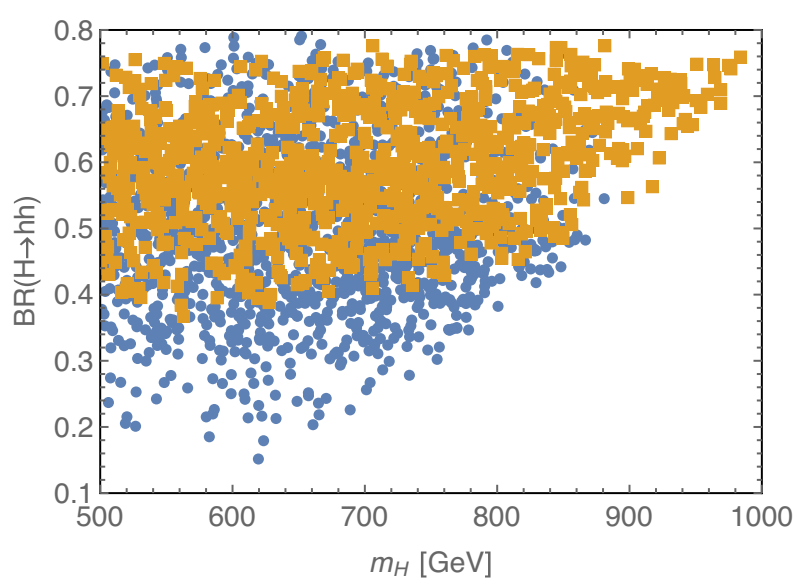

(a)

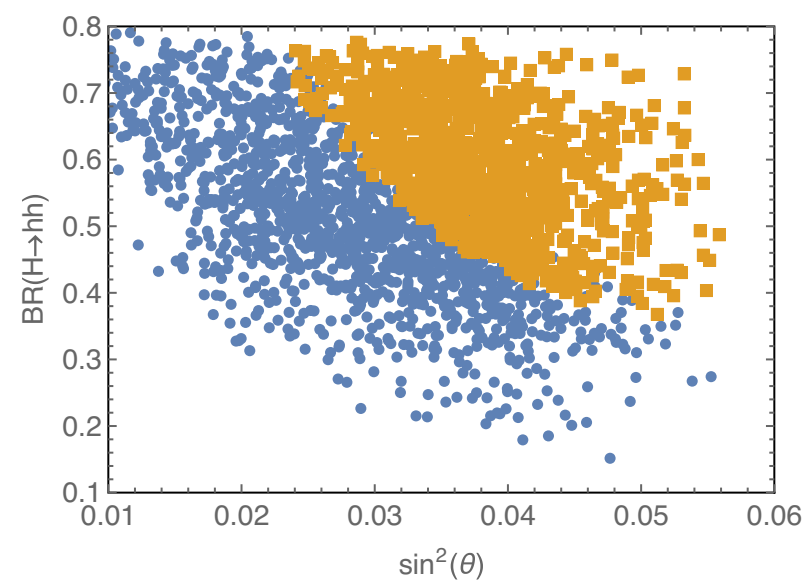

(b)

FIG. 4. 95\% confidence level constraints interpreted in the singlet scenario of Sec. II. (a) Constraints from gluon fusion in blue dots and from $h h+2 j$ in orange squares as a function of the mass and branching fraction of $H \rightarrow h h$. (b) Similar to (a), but we show the correlation of $\sin ^{2} \theta$ with the $H \rightarrow h h$ branching.

at $m_{H} \sim 580 \mathrm{GeV}$ arises from the two distinct strategies separating low and high mass resonances.

We observe that the double Higgs resonant search in the VBF mode can significantly contribute to the heavy Higgs resonant analyses. The increase in the ratio $\sigma_{\mathrm{VBF}} / \sigma_{\mathrm{GF}}$ for larger $m_{H}$ leads to comparable sensitivities between the VBF and GF channels for $m_{H} \sim 900 \mathrm{GeV}$. Whereas the VBF search displays stronger limits at the high $m_{H}$ regime, it can also contribute to further constrain the low mass scenarios $500 \mathrm{GeV}<m_{H}<900 \mathrm{GeV}$ via a combination between the GF and VBF analyses.

In order to understand the relevance of the GF and VBF limits on the singlet-extension scenario discussed in Sec. II, we interpret the constraints in the aforesaid model. We scan over the singlet model parameter space for $\left|\lambda_{i}\right| \leq 4 \pi$ and include the $W$ mass constraint from Refs. [51,53] as it typically imposes the strongest constraint on the model's parameter space. The results are shown in Fig. 4. The constraints from gluon fusion $g g \rightarrow h h$ are displayed in blue points, while those of $p p \rightarrow h h j j$ are given in orange squares. We see that the vector boson fusion provides significant sensitivity for higher masses where the gluon fusion projection becomes insensitive.

While there is a region where gluon fusion and VBF overlap and can be used to further hone the LHC sensitivity to this scenario through a statistical combination, we also see regions in the branching ratio $H \rightarrow h h$ where VBF provides genuine, new sensitivity that cannot be accessed with the gluon fusion analysis. This region is characterized by $125 \mathrm{GeV}$ Higgs boson signal strength modifiers of $\lesssim 4 \%$. Given the HL-LHC projections of Ref. [75], this suggests that the resonance search in the WBF channel can also explore the model's parameter space beyond the precision that can be obtained from $125 \mathrm{GeV}$ signal studies.

QCD contributions to $p p \rightarrow h h j j$ are not the dominant contribution in this mass region (it is a sizable contribution for the theoretical interpretation of the results of Ref. [19]), it nonetheless is sizable and should be included in investigations possibly as a separate signal contribution to enable a consistent theoretical interpretation.

\section{SUMMARY AND CONCLUSIONS}

Weak boson fusion through its distinct phenomenological properties provides a unique opportunity for new physics searches. In scenarios with isospin singlet mixing, decays of a heavy Higgs partner into $125 \mathrm{GeV}$ Higgs bosons can be preferred while more obvious decays into top quarks suffer from interference distortion [47], and decays into massive weak bosons might be less dominant. Given that the weak boson fusion production cross section becomes comparable to a gluon fusion cross section for SM-like production at around $1 \mathrm{TeV}$, the $\mathrm{WBF}$ production at small mixing angles becomes a phenomenologically relevant channel. In this paper, we investigate the WBF production of heavy Higgs partners with subsequent decay $H \rightarrow h h$. We show that this channel, which has been somewhat overlooked in the past, provides additional relevant new physics potential. In parallel, we show that the gluon fusion component of the vector boson fusion channel remains sizeable and should be included in experimental analysis to enable a consistent theoretical interpretation of reported results.

\section{ACKNOWLEDGMENTS}

We thank Stephen Brown and Peter Galler for helpful conversations. C. E. is supported by the UK Science and Technology Facilities Council (STFC) under Grant No. ST/ P000746/1. C. E. also acknowledges support through the IPPP associate scheme. D. G. was supported by the U.S. Department of Energy under Grant No. DE-SC 0016013. M. S. is supported by the STFC under Grant No. ST/ P001246/1. 
[1] S. Dawson, S. Dittmaier, and M. Spira, Phys. Rev. D 58, 115012 (1998).

[2] R. Frederix, S. Frixione, V. Hirschi, F. Maltoni, O. Mattelaer, P. Torrielli, E. Vryonidou, and M. Zaro, Phys. Lett. B 732, 142 (2014).

[3] S. Borowka, N. Greiner, G. Heinrich, S. P. Jones, M. Kerner, J. Schlenk, U. Schubert, and T. Zirke, Phys. Rev. Lett. 117, 012001 (2016); 117, 079901(E) (2016).

[4] D. de Florian et al. (LHC Higgs Cross Section Working Group), arXiv:1610.07922.

[5] M. Grazzini, G. Heinrich, S. Jones, S. Kallweit, M. Kerner, J. M. Lindert, and J. Mazzitelli, J. High Energy Phys. 05 (2018) 059.

[6] J. Alison et al., in Proceedings of Double Higgs Production at Colliders, Batavia, IL, USA, 2019, edited by B. Di Micco, M. Gouzevitch, J. Mazzitelli, and C. Vernieri [https://lss.fnal .gov/archive/2019/conf/fermilab-conf-19-468-e-t.pdf].

[7] J. Baglio, F. Campanario, S. Glaus, M. Mhlleitner, J. Ronca, M. Spira, and J. Streicher, J. High Energy Phys. 04 (2020) 181.

[8] T. Plehn and M. Rauch, Phys. Rev. D 72, 053008 (2005).

[9] A. Papaefstathiou and K. Sakurai, J. High Energy Phys. 02 (2016) 006.

[10] M. Chiesa, F. Maltoni, L. Mantani, B. Mele, F. Piccinini, and X. Zhao, arXiv:2003.13628.

[11] R. N. Cahn and S. Dawson, Phys. Lett. 136B, 196 (1984); 138B, 464(E) (1984).

[12] D. L. Rainwater, D. Zeppenfeld, and K. Hagiwara, Phys. Rev. D 59, 014037 (1998).

[13] D. L. Rainwater and D. Zeppenfeld, Phys. Rev. D 60, 113004 (1999); 61, 099901(E) (2000).

[14] T. Plehn, D. L. Rainwater, and D. Zeppenfeld, Phys. Rev. D 61, 093005 (2000).

[15] M. J. Dolan, C. Englert, N. Greiner, K. Nordstrom, and M. Spannowsky, Eur. Phys. J. C 75, 387 (2015).

[16] F. Bishara, R. Contino, and J. Rojo, Eur. Phys. J. C 77, 481 (2017).

[17] E. Arganda, C. Garcia-Garcia, and M. J. Herrero, Nucl. Phys. B945, 114687 (2019).

[18] M. J. Dolan, C. Englert, N. Greiner, and M. Spannowsky, Phys. Rev. Lett. 112, 101802 (2014).

[19] G. Aad et al. (ATLAS Collaboration), J. High Energy Phys. 07 (2020) 108.

[20] V. Del Duca, W. Kilgore, C. Oleari, C. Schmidt, and D. Zeppenfeld, Phys. Rev. Lett. 87, 122001 (2001).

[21] V. Del Duca, W. Kilgore, C. Oleari, C. R. Schmidt, and D. Zeppenfeld, Phys. Rev. D 67, 073003 (2003).

[22] V. del Duca, G. Klmke, M. Moretti, F. Piccinini, R. Pittau, A. D. Polosa, and D. Zeppenfeld, in Proceedings of the 42nd Rencontres de Moriond on QCD and High Energy Hadronic Interactions: La Thuile, Italy, 2007 (Gioi Publishers, Hanoi, 2007), pp. 205-208.

[23] V. Del Duca, G. Klamke, D. Zeppenfeld, M. L. Mangano, M. Moretti, F. Piccinini, R. Pittau, and A. D. Polosa, J. High Energy Phys. 10 (2006) 016.

[24] B. Grzadkowski, H. E. Haber, O. M. Ogreid, and P. Osland, J. High Energy Phys. 12 (2018) 056.

[25] D. Fontes, M. Mhlleitner, J. C. Romo, R. Santos, J. P. Silva, and J. Wittbrodt, J. High Energy Phys. 02 (2018) 073.

[26] H. Georgi and M. Machacek, Nucl. Phys. B262, 463 (1985).
[27] J. F. Gunion, R. Vega, and J. Wudka, Phys. Rev. D 42, 1673 (1990).

[28] K. Hartling, K. Kumar, and H. E. Logan, Phys. Rev. D 91, 015013 (2015).

[29] J. F. Gunion, R. Vega, and J. Wudka, Phys. Rev. D 43, 2322 (1991).

[30] G. Ferretti, J. High Energy Phys. 06 (2014) 142.

[31] M. Golterman and Y. Shamir, Phys. Rev. D 91, 094506 (2015).

[32] S. Godfrey and K. Moats, Phys. Rev. D 81, 075026 (2010).

[33] K. Cheung and D. K. Ghosh, J. High Energy Phys. 11 (2002) 048.

[34] C. Englert, E. Re, and M. Spannowsky, Phys. Rev. D 88, 035024 (2013).

[35] M. Zaro and H. Logan, 2015, https://cds.cern.ch/record/ 2002500.

[36] C. Degrande, K. Hartling, H. E. Logan, A. D. Peterson, and M. Zaro, Phys. Rev. D 93, 035004 (2016).

[37] T. Binoth and J. J. van der Bij, Z. Phys. C 75, 17 (1997).

[38] S. Dittmaier et al. (LHC Higgs Cross Section Working Group), arXiv:1101.0593.

[39] D. Das, Phys. Rev. D 99, 095035 (2019).

[40] K. J. F. Gaemers and F. Hoogeveen, Phys. Lett. 146B, 347 (1984).

[41] D. Dicus, A. Stange, and S. Willenbrock, Phys. Lett. B 333, 126 (1994).

[42] R. Frederix and F. Maltoni, J. High Energy Phys. 01 (2009) 047.

[43] M. Carena and Z. Liu, J. High Energy Phys. 11 (2016) 159.

[44] B. Hespel, F. Maltoni, and E. Vryonidou, J. High Energy Phys. 10 (2016) 016.

[45] D. Buarque Franzosi, F. Fabbri, and S. Schumann, J. High Energy Phys. 03 (2018) 022.

[46] C. Englert, P. Galler, and C. D. White, Phys. Rev. D 101, 035035 (2020).

[47] P. Basler, S. Dawson, C. Englert, and M. Mhlleitner, Phys. Rev. D 101, 015019 (2020).

[48] C.-Y. Chen, S. Dawson, and I. M. Lewis, Phys. Rev. D 91, 035015 (2015).

[49] F. Bojarski, G. Chalons, D. Lopez-Val, and T. Robens, J. High Energy Phys. 02 (2016) 147.

[50] S. Dawson and M. Sullivan, Phys. Rev. D 97, 015022 (2018).

[51] D. Lpez-Val and T. Robens, Phys. Rev. D 90, 114018 (2014).

[52] A. Falkowski, C. Gross, and O. Lebedev, J. High Energy Phys. 05 (2015) 057.

[53] T. Robens and T. Stefaniak, Eur. Phys. J. C 75, 104 (2015).

[54] K. Arnold et al., Comput. Phys. Commun. 180, 1661 (2009).

[55] J. Alwall, R. Frederix, S. Frixione, V. Hirschi, F. Maltoni, O. Mattelaer, H. S. Shao, T. Stelzer, P. Torrielli, and M. Zaro, J. High Energy Phys. 07 (2014) 079.

[56] T. Sjostrand, S. Mrenna, and P. Z. Skands, Comput. Phys. Commun. 178, 852 (2008).

[57] M. Cacciari, G. P. Salam, and G. Soyez, Eur. Phys. J. C 72, 1896 (2012).

[58] Proceedings of the 6th International Conference on Physics in Collision, Chicago, 1986, edited by M. Derrick (World Scientific, Singapore, 1987), p. 542, https://inspirehep.net/ literature/257683. 
[59] J. D. Bjorken, Phys. Rev. D 47, 101 (1993).

[60] V. D. Barger, K.-m. Cheung, T. Han, and D. Zeppenfeld, Phys. Rev. D 44, 2701 (1991); 48, 5444(E) (1993).

[61] V. D. Barger, R. J. N. Phillips, and D. Zeppenfeld, Phys. Lett. B 346, 106 (1995).

[62] S. Dawson, Nucl. Phys. B249, 42 (1985).

[63] T. Figy, V. Hankele, and D. Zeppenfeld, J. High Energy Phys. 02 (2008) 076.

[64] D. Goncalves, T. Plehn, and J. M. Thompson, Phys. Rev. D 95, 095011 (2017).

[65] A. M. Sirunyan et al. (CMS Collaboration), J. High Energy Phys. 08 (2018) 152.

[66] M. Aaboud et al. (ATLAS Collaboration), J. High Energy Phys. 01 (2019) 030.
[67] M. Aaboud et al. (ATLAS Collaboration), Phys. Lett. B 784, 173 (2018).

[68] G. Aad et al. (ATLAS Collaboration), arXiv:2005.05138.

[69] F. Buccioni, S. Kallweit, S. Pozzorini, and M. F. Zoller, J. High Energy Phys. 12 (2019) 015.

[70] T. Figy, C. Oleari, and D. Zeppenfeld, Phys. Rev. D 68, 073005 (2003).

[71] J. M. Campbell, R. K. Ellis, and G. Zanderighi, J. High Energy Phys. 10 (2006) 028.

[72] J. Campbell and T. Neumann, J. High Energy Phys. 12 (2019) 034.

[73] A. L. Read, J. Phys. G 28, 2693 (2002).

[74] J. R. Andersen et al. (LHC Higgs Cross Section Working Group), arXiv:1307.1347.

[75] J. de Blas et al., J. High Energy Phys. 01 (2020) 139. 